\title{
Selective Laser Melting Process of Al-Based Pyramidal Horns for the W-Band: Fabrication and Testing
}

\author{
L. Lamagna $^{1}$ (D) A. Paiella ${ }^{1} \cdot$ S. Masi ${ }^{1} \cdot$ L. Bottini $^{2} \cdot$ A. Boschetto $^{2} \cdot$ F. Veniali $^{2}$
}

Received: 25 May 2020 / Accepted: 24 November 2020 / Published online: 8 January 2021

(C) The Author(s) 2021

\begin{abstract}
In the context of exploring the possibility of using Al-powder Selective Laser Melting to fabricate horn antennas for astronomical applications at millimeter wavelengths, we describe the design, the fabrication, the mechanical characterization, and the electromagnetic performance of additive manufactured horn antennas for the Wband. Our aim, in particular, is to evaluate the performance impact of two basic kinds of surface post-processing (manual grinding and sand-blasting) to deal with the well-known issue of high surface roughness in 3D printed devices. We performed comparative tests of co-polar and cross-polar angular response across the whole $\mathrm{W}$ band, assuming a commercially available rectangular horn antenna as a reference. Based on gain and directivity measurements of the manufactured samples, we find decibel-level detectable deviations from the behavior of the reference horn antenna, and marginal evidence of performance degradation at the top edge of the W-band. We conclude that both kinds of post-processing allow achieving good performance for the W-band, but the higher reliability and uniformity of the sand-blasting postprocess encourage exploring similar techniques for further development of aluminum devices at these frequencies.
\end{abstract}

Keywords Additive manufacturing · Horn antennas

\section{Introduction}

Additive manufacturing (AM) is a host of technologies able to fabricate very intricate geometrical parts directly from a virtual model without the need of specific tools or

L. Lamagna

luca.lamagna@roma1.infn.it

1 Dipartimento di Fisica, Sapienza Università di Roma, P.le A. Moro 2, 00185 Roma, Italy

2 Dipartimento di Ingegneria Meccanica e Aerospaziale, Sapienza Università di Roma, Via Eudossiana 18, 00184 Roma, Italy 
dies [2]. Its flexibility in terms of materials (polymers, metals, ceramics) and realizable geometries makes these technologies suitable for a large number of applications (visual and functional prototypes, patterns for metal casting, tools and molds, end parts for short run and medium series production) in different fields (automotive, aerospace, biomedical, etc.) [28].

One of the emerging potential applications in the last years concerns the realization of horn antennas and waveguide components suitable for operation in microwaves, spanning telecommunications, navigation, remote-sensing, and astronomy. In these fields, application-specific designs are commonly employed to optimize system performance and a comparatively high level of customization can be necessary to achieve the best results. On the other hand, the procurement of highly cutomized microwave components through commercial supply channels often results from the trade-off between off-the-shelf availability, customization and development timescales offered by microwave companies, and actual supply volume. This has an impact in particular on the research applications, where instrumentation is rarely mass-produced and cannot always rely on dedicated and cost-effective production efforts from the manufacturers.

AM not only requires careful adaptation of the overall workflow from design to testing of the final part with respect to older, more consolidated techniques, but it also offers the valuable opportunity to mitigate some of the abovementioned issues. In order to effectively manufacture a passive microwave component, it is necessary to rely on a process with compliant mechanical tolerance (typical constraints are of the order of $1 / 20$ of the shortest operating wavelengths) and electromagnetic performance consistent with the foreseen application of the device. Satisfactory results have been reported up to hundreds of gigahertz for electroplated dielectric-based technologies (like fused deposition modeling, stereolithography, binder jetting) where the needed surface conductance is achieved after 3D printing, through a separate process involving the deposition of conductive material on the surfaces [15]. Alternatively, all-metal techniques like selective laser melting (SLM) allow manufacture of conductive parts directly in a single-step process. SLM, as defined by [25], is a powder bed fusion process where a laser beam fully melts powders aiming to create solid parts after cooldown. The process consists in the iteration of a few steps like in other AM technologies:

- A first powder layer is spread on the building platform by a scraper;

- A scanning mirror redirects the laser source in order to scan and fuse a selected area of the powder bed according to the digital slices of the 3D model;

- A piston lowers down the base plate and the previous step is repeated until the part is completely fabricated [13].

The process is characterized by a rapid cooling, leading to a marked buildup of residual stresses which require a thermal treatment after the fabrication. This technology offers several advantages: near-net-shape complex part geometry, full-density metallic material, good mechanical and electrical properties, low material wastage, and high-production flexibility compared to conventional AM processes. These benefits well meet the needs for the fabrication of horn antennas, which must feature high electrical conductance, high corrosion resistance, and free-form geometry. A major 
concern about this technology is related to the surface roughness of the manufactured parts and to its impact on their electromagnetic performance at the wavelengths of interest. Hence, the fabrication must be accompanied by a proper selection of the SLM process parameters and of the post-processing steps. In addition, design adaptations must be expected and carefully implemented in order to overcome the restrictions of the process workflow: for example, proper supporting structures must often be added to the functional section of the design, in order to prevent the collapse of the unfinished part during the manufacturing and to allow an efficient and uniform heat dissipation after the melting of the powder layers.

Excellent millimeter-wave performance of SLM-fabricated conical horn antennas and waveguides has been demonstrated above $300 \mathrm{GHz}$ for processes based on $\mathrm{Cu}$ $15 \mathrm{Sn}$ powder $[29,30]$. This material is clearly attractive by the points of view of the electrical conductivity and of the effectiveness of the post-processing in mitigating the surface roughness. On the other hand, it is also prone to intrinsic limitations in view of applications in millimeter-wave astronomy: $\mathrm{Cu}$-based components are comparatively heavy and therefore not easily implemented in the large-format cryogenic focal planes of millimeter-wave telescopes, which often feature close-packings of hundreds to thousands of such devices. For these applications, the preference shifts toward lighter materials (such as aluminum) especially if instruments are hosted on balloon-borne platforms $[16,21]$, where weight containment is a key factor in payload design. More importantly, modern detector technologies for millimeter-wave astronomy rely on superconducting cryogenic sensors operated at temperatures of a few hundreds of $\mathrm{mK}$, for which the readout is often sensitive to external magnetic fields and highly benefit from magnetic shielding by superconducting housings and enclosures. Aluminum, with a bulk critical temperature of $1.2 \mathrm{~K}$, allows implementing at least one stage of magnetic shielding at no additional cost, while directly shaping it in the form of the quasi-optical components (horns, waveguides, orthomode transducers) that couple the detectors to radiation $[8,9,14,17,18]$. Over the past few years, the performance of SLM-fabricated, Al-based components such as resonant cavities, waveguide filters, custom-designed horns, and ortho-mode transducers has been convincingly demonstrated for microwave frequencies up to few tens of gigahertz and various post-processing workflows from the raw part to the final component have been explored and proved, even for complex integrated assemblies (see, e.g., $[6,19,20]$ ). Recently, such studies have been pushed up to the $K_{a}$-band [1], but an investigation of the same processes with similar devices and even higher frequencies is still desirable. In this work, for the first time, we take a step in this direction: we designed and fabricated a simple pyramidal horn + waveguide assembly suitable for single-mode operation in the W-band. Two samples were postprocessed with different techniques to mitigate the surface roughness, one through sand-blasting and the other one through manual grinding, and their W-band performance was measured and compared with that of a reference, commercially available horn with the same geometry. This paper is organized as follows: in Section 2, we describe the design of the horns and the necessary adaptations to make it compliant with the AM technology; in Section 3, we highlight the details of the SLM process and the specific process parameters used for fabrication; in Section 4, we show the surface characterization of the samples after the application of the two different kinds 
of post-processing; in Section 5, we describe the electromagnetic performance test setup and highlight the rationale of our performance measurements; in Section 6, we highlight the main results and compare them with similar data available in the literature. We present our conclusions in Section 7.

\section{Horn Design}

The horn has been designed in order to reproduce the features of the commercially available Aerowave 08-7025 horn. Its geometry is sketched in the left panel of Fig. 1.

The design was customized to reproduce an integrated horn-waveguide assembly, with the horn feeding a standard WR8 rectangular waveguide. The interface flange needed to couple the assembly to the test setup was built via standard subtractive techniques and integrated with the samples after fabrication. This choice has allowed reducing the excess insertion loss into the waveguide, which could result from unoptimized tolerancing and finishing of an additively fabricated interface flange.

The SLM fabrication needs several steps: part design, conversion to Standard Triangulation Language (STL), part orientation and support generation, setting of processing parameters, physical fabrication, and post-processing.

The solid model has been developed in a commercial computer-aided design. The STL file, which is necessary for the subsequent processing and for the machine software input, has been generated by taking care of the typical errors which can prevent
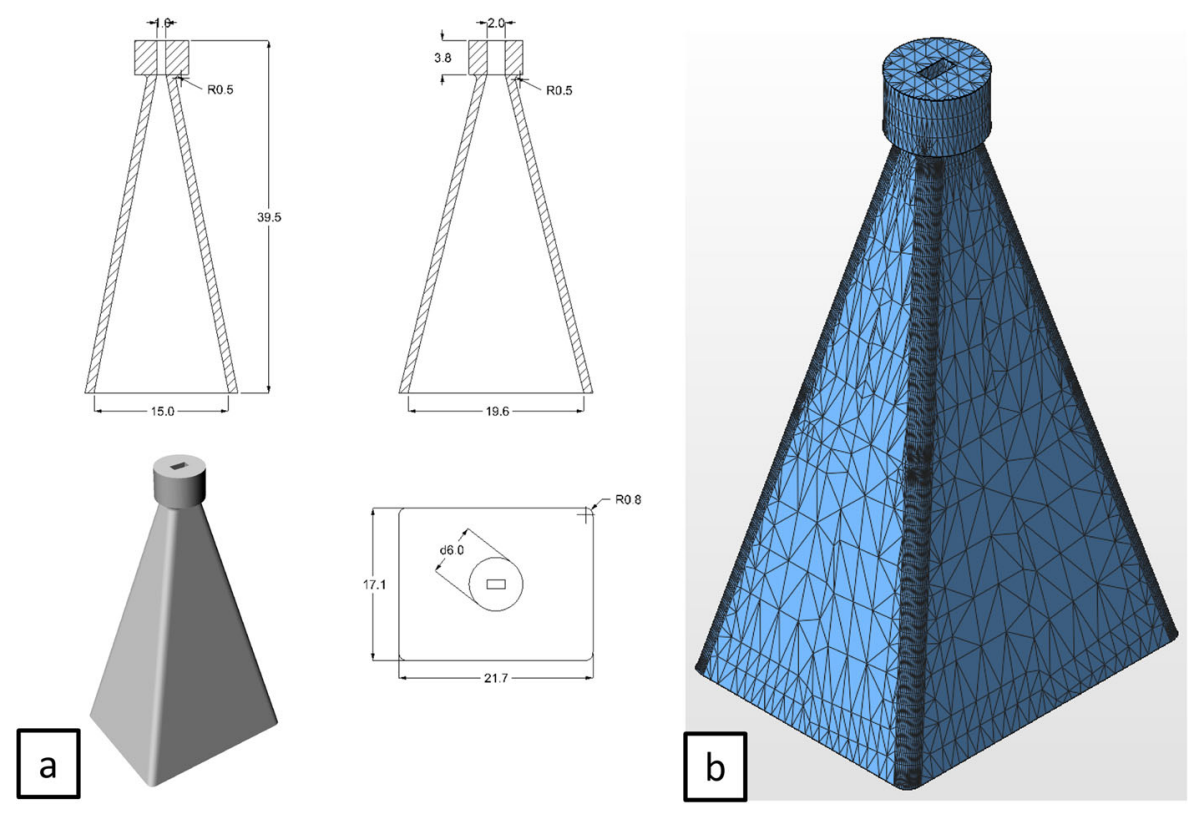

Fig. 1 Left panel Drawing and 3D virtual model. Right panel STL file of the designed horn 
the AM fabrication or locally modify the part geometry [7]: for this purpose, the maximum aspect ratio of each triangle has been limited to 2 , in order to strengthen the slicing operation and the chordal error has been set to $0.005 \mathrm{~mm}$ which is negligible with respect to the expected SLM accuracy $( \pm 0.1 \mathrm{~mm}$ for aluminum powder as reported on the EOS datasheet).

The output, shown in the right panel of Fig. 1, is characterized by 14,060 triangles and 21,090 edges. It has been analyzed and verified through Autodesk Netfabb 2017 to ensure that no errors in terms of border edges, invalid orientation, and holes were found.

\section{Horn Fabrication}

The designed horn has been fabricated through the SLM process. The employed machine is the EOSINT ${ }^{\circledR}$ M290 equipped with a 400-W ytterbium fiber continuum laser. The laser beam has a Gaussian intensity profile with a $2 \sigma$ diameter of $100 \mathrm{~mm}$. The employed material is the AlSi10Mg gas atomized powder provided by EOS Gmbh. It is a casting Al-Si alloy with the nominal composition reported in Table 1.

The powder has been characterized by means of equivalent diameter distribution through digital image processing of SEM pictures. In Fig. 2, the distribution by volume of the employed powder equivalent diameter is shown. In order to synthetically characterize this distribution, we define $d_{X}$ as the diameter value at which $X \%$ of the sample's volume is characterized by particles with diameters less than this value. The distribution span, considered as the difference between $d_{10}$ and $d_{90}$, is in the range [16.2 $\mathrm{mm}, 39.6 \mathrm{~mm}$ ]; in addition, the distribution is almost symmetrical since the median $d_{50}$ and the averages are very close (28.9 mm and $29.4 \mathrm{~mm}$, respectively).

This alloy is characterized by good thermal properties, low weight, good strength, hardness, dynamic properties, and low electrical resistivity as shown in Table 2, where the values after the SLM process are reported [11]. Mechanical tests have been performed using proportional specimens $5 \mathrm{~mm}$ in diameter according to [26]. It is

Table 1 Composition of AlSi10Mg powder

\begin{tabular}{ll}
\hline Element & Weight $\%$ \\
\hline $\mathrm{Si}$ & $9.0-11$ \\
$\mathrm{Fe}$ & $\leq 0.55$ \\
$\mathrm{Cu}$ & $\leq 0.05$ \\
$\mathrm{Mn}$ & $\leq 0.45$ \\
$\mathrm{Mg}$ & $0.20-0.45$ \\
$\mathrm{Ni}$ & $\leq 0.05$ \\
$\mathrm{Zn}$ & $\leq 0.10$ \\
$\mathrm{~Pb}$ & $\leq 0.05$ \\
$\mathrm{Sn}$ & $\leq 0.05$ \\
$\mathrm{Ti}$ & $\leq 0.15$ \\
$\mathrm{Al}$ & Balance \\
\hline
\end{tabular}




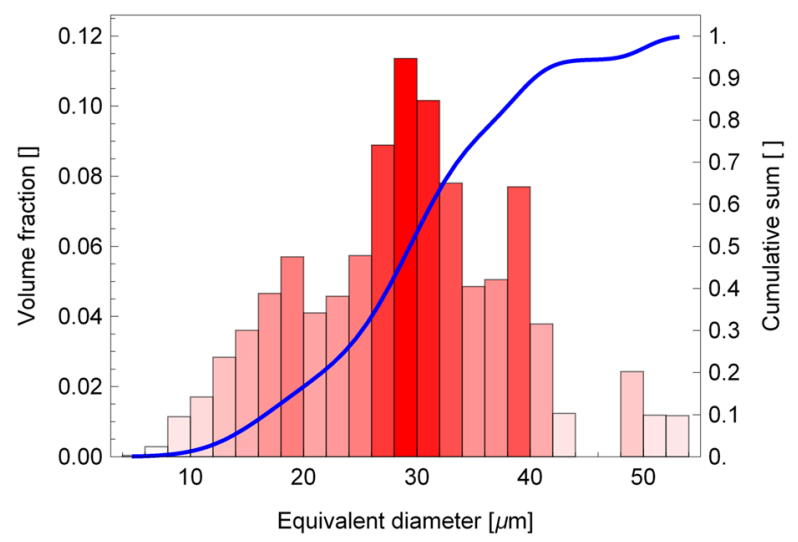

Fig. 2 Equivalent diameter volume fraction (histogram in red) and cumulative sum (blue line) distributions

noticeable that the mechanical and thermal properties exhibit a slight variation when measured along directions parallel and perpendicular to the plane of the building platform.

The employed process parameters are listed in Table 3 and correspond to a volumetric specific energy of $49.9 \mathrm{~J} / \mathrm{mm}^{3}$ : this high energy allowed overcoming some of the issues related to the SLM processing of AlSi10Mg, such as the low absorptivity and high reflectivity [5] and the incorporation of powder oxides into the molten metal [4].

The used scanning strategy was of the island stripe type. The laser path is composed of subsequent segments with a length $S_{w}$ and an overlapping $S_{o}$ : the short length is necessary to reduce distortions inside the layer caused by residual stresses; the overlapping is required to avoid discontinuity. The distance between the lines

Table 2 SLM-manufactured material properties

\begin{tabular}{ll}
\hline Tensile strength (horizontal and vertical) & $(460 \pm 20) \mathrm{MPa}$ \\
Yield strength (horizontal) & $(270 \pm 10) \mathrm{MPa}$ \\
Yield strength (vertical) & $(240 \pm 10) \mathrm{MPa}$ \\
Modulus of elasticity (horizontal) & $(75 \pm 10) \mathrm{GPa}$ \\
Modulus of elasticity (vertical) & $(70 \pm 10) \mathrm{GPa}$ \\
Elongation at break (horizontal) & $(9 \pm 2) \%$ \\
Elongation at break (vertical) & $(6 \pm 2) \%$ \\
Hardness & $(119 \pm 5) \mathrm{HB}$ \\
Fatigue strength (vertical) & $(97 \pm 7) \mathrm{MPa}$ \\
Thermal conductivity (horizontal) & $(103 \pm 5) \mathrm{W} /(\mathrm{m} \mathrm{K})$ \\
Thermal conductivity (vertical) & $(119 \pm 5) \mathrm{W} /(\mathrm{m} \mathrm{K})$ \\
Specific heat capacity (horizontal) & $(920 \pm 50) \mathrm{J} /(\mathrm{kg} \mathrm{K})$ \\
Specific heat capacity (vertical) & $(910 \pm 50) \mathrm{J} /(\mathrm{kg} \mathrm{K})$ \\
Electrical resistivity & $7.78 \mu \Omega \mathrm{cm}$ \\
\hline
\end{tabular}


Table 3 Used SLM process parameters

\begin{tabular}{ll}
\hline Power & $370 \mathrm{~W}$ \\
Scan speed & $1300 \mathrm{~mm} / \mathrm{s}$ \\
Hatch distance $H_{d}$ & $0.19 \mathrm{~mm}$ \\
Stripe length $S_{w}$ & $7 \mathrm{~mm}$ \\
Stripe overlap $S_{o}$ & $0.02 \mathrm{~mm}$
\end{tabular}

is called hatch distance $H_{d}$. Moreover, on each layer, the direction of the scanning was rotated by $67^{\circ}$ between consecutive layers in order to reduce residual stresses between the layers. The chosen building orientation is reported in Fig. 3: the horn main axis is parallel to the stratification direction and the part does not increase in size against the recoating direction, thus reducing the force engaging with the obstacle. In this way, the support structures are necessary only between the building platform and the part and no other surfaces are involved. Particular attention has been paid to the support type: solid structures have been designed in order to avoid local detachment of the part from the platform during the fabrication.

After the fabrication, a thermal treatment of $2 \mathrm{~h}$ at $300{ }^{\circ} \mathrm{C}$ was performed in order to release the built-up stresses and avoid deformations during the detachment from the building platform. The detachment was performed through abrasive metallographic cutting. Particular care has been taken in order to limit the part deformation due to the cutting forces: the cutting speed and the feed have been set to $40 \mathrm{~m} / \mathrm{s}$ and $0.02 \mathrm{~mm} /$ round, respectively, leading to a maximum cutting force of $0.5 \mathrm{~N}$. Two samples built with the same process parameters were subsequently conditioned with the two abovementioned techniques (sand-blasting and manual grinding) to eliminate exceeding powders and mitigate the surface roughness. It is worthy to note that none of the two techniques is expected to yield significant gain in the surface quality of the waveguide section due to its small size. Therefore, a level of loss due to the residual roughness of this section is still expected and any improvement with respect to the unfinished part should be attributed to the quality of the horn surfaces.

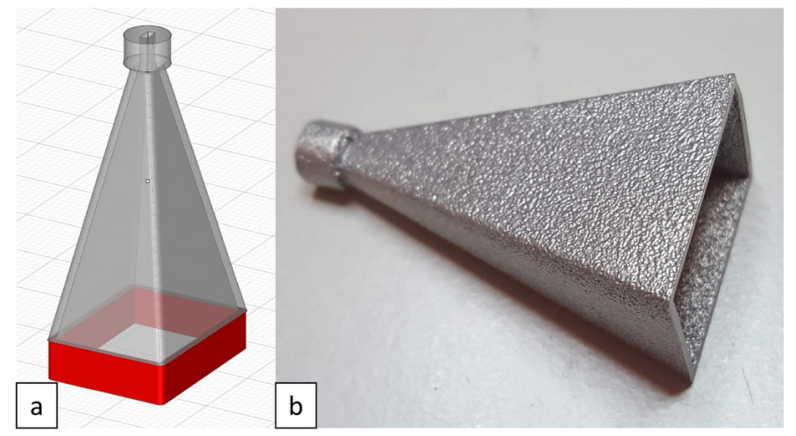

Fig. 3 Left panel Building orientation and support structures. Right panel Fabricated horn right after the SLM processing 


\section{Surface Characterization}

The surface roughness of SLM parts is markedly influenced by the process parameters and by the building orientation. In a previous work [3], the attainable roughness of a surface fabricated through SLM under the abovementioned conditions has been modelled. According to Eq. 1, the average roughness of the functional surfaces can be predicted by considering the horn orientation with respect to the local stratification angle $\alpha$, i.e., the angle between the stratification direction and the normal to the surface. Hence, the internal vertical walls (waveguide section) and the internal inclined surfaces (horn section) are characterized by $90^{\circ}$ and $105^{\circ}$ local stratification angles, respectively. For these surfaces, an average roughness $R_{a}$ of about $24 \mu \mathrm{m}$ is predicted over a measurement length pair to $e_{1}+e_{2}$.

$$
\begin{aligned}
R_{a} & =\frac{1}{e_{1}+e_{2}}\left(\int_{-e_{1}}^{0}\left|x \cot \alpha+30 \cos \alpha+20 \sin ^{2} \frac{x \pi}{30}-m\right| d x+\right. \\
& +\int_{0}^{e_{2}} \mid 30 \cos \alpha-x \tan \alpha+\left\{\begin{array}{l}
75 \sin ^{6}(x \pi / 60) \quad x<60 \\
20 \sin ^{2}(x \pi / 60) \quad x \geq 60
\end{array}-m \mid d x\right),
\end{aligned}
$$

where

$$
\begin{aligned}
m & =\frac{1}{e_{1}+e_{2}}\left[\int_{-e_{1}}^{0}\left(x \cot \alpha+30 \cos \alpha+20 \sin ^{2} \frac{x \pi}{30}\right) d x+\right. \\
& +\int_{0}^{e_{2}}\left(30 \cos \alpha-x \tan \alpha+\left\{\begin{array}{ll}
75 \sin ^{6}(x \pi / 60) & x<60 \\
20 \sin ^{2}(x \pi / 60) & x \geq 60
\end{array}\right) d x\right],
\end{aligned}
$$

The surface roughness of the internal horn has been measured through a Mitutoyo SJ412 equipped with a stylus characterized by a 180 -mm-long and $2-\mu \mathrm{m}$-radius diamond tip. According to [22], the sampling length and the evaluation length have been set to $2.5 \mathrm{~mm}$ and $12.5 \mathrm{~mm}$ respectively. A spline profile filter, according to [24], has been applied to determine the roughness contribution to the profile: the cutoff and the short wavelength cutoff have been set to $2.5 \mathrm{~mm}$ and $8 \mu \mathrm{m}$ respectively. The roughness parameters have been calculated according to [23].

The profile of the horn internal surface is shown in the left panels of Fig. 4 for the two samples. The profile of the sand-blasted horn shows smoothed high peaks and deep valleys according to the expected SLM morphology. The distribution of the asperities is quite symmetrical as highlighted by the Abbott-Firestone curve [3], which describes the cumulative distribution of the asperities. In the manual-grinded sample, a suppression of the positive peaks is visible, but many of the deep valleys are still present. This is confirmed by the Abbott-Firestone curve which shows that more than $60 \%$ of the profile is above the zero level. Moreover, some additional defects are present, as expected as a consequence of any grinding operation.

As a quantification of these observations, a proper set of roughness parameters has been chosen, following the definitions in [12]. The results are shown in Table 4. A marked decreasing on $R_{a}$ is achieved by the secondary finishing but it is well known that this parameter is not sufficient to describe surface morphology [27]. By taking the $R_{p}$ and $R_{v}$ parameters, which measure the average height of the peaks and the valleys respectively, the previous assessment is confirmed: the reduction of 

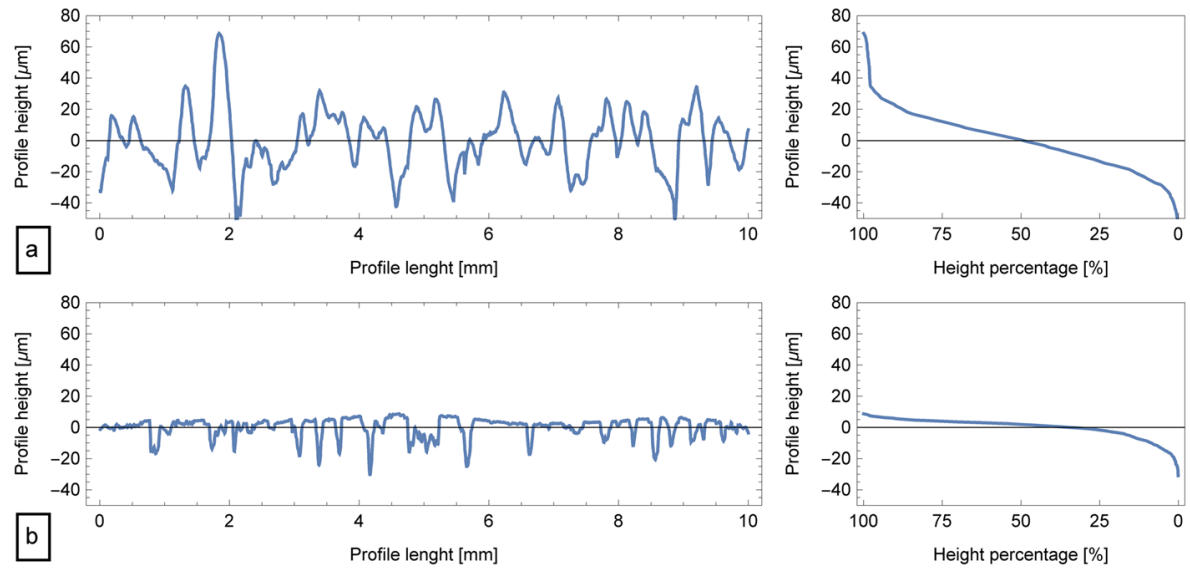

Fig. 4 Sand-blasted (top panel) and manual-grinded (bottom panel) roughness profiles (left panels) and corresponding Abbott-Firestone curves (right panels)

the peaks is $84 \%$, conversely the valleys are reduced by $50 \%$. The surface coming directly from the SLM fabrication is symmetrical; in fact, the $R_{s k}$ is about 0 and it shows a Gaussian behavior confirmed by the $R_{k u}$ about 3 which is the exact value for the normal distribution. After the secondary operation, the distribution moves to the top ( $R_{s k}$ is negative) and a pronounced peakedness is observed $\left(R_{k u}>5\right)$. For what concerns the spacing, a worsening of the profile is measured because the original spacing pair to $800 \mu \mathrm{m}$ is reduced to $575 \mu \mathrm{m}$ : this highlights an increment of the profile defects. Accordingly, the root mean square of the profile slope shows a slight reduction from 0.256 to 0.201 , notwithstanding the marked reduction in $R_{a}$. This parameter is often used in mechanical and optical science to understand the reflectivity of a surface.

Other interesting information can be provided by considering the unfiltered profiles. In Fig. 5 the sand blasted horn shows a quite flat trend. After the grinding the profile is markedly curved highlighting a non-uniform finishing. This issue must well be considered in the selection of the finishing processing parameters and the effects upon the horn frequency response.

Table 4 Roughness parameters of sand-blasted and manual-grinded horns

\begin{tabular}{lll}
\hline Horn & Sand-blasted & Manual-grinded \\
\hline$R_{a}(\mu \mathrm{m})$ & 14.86 & 5.54 \\
$R_{p}(\mu \mathrm{m})$ & 41.52 & 6.82 \\
$R_{v}(\mu \mathrm{m})$ & 47.41 & 23.51 \\
$R_{s k}$ & -0.043 & -1.635 \\
$R_{k u}$ & 2.882 & 5.525 \\
$R_{S M}(\mu \mathrm{m})$ & 799.6 & 575.1 \\
$R_{\Delta M}(\mu \mathrm{m} / \mu \mathrm{m})$ & 0.256 & 0.201 \\
\hline
\end{tabular}



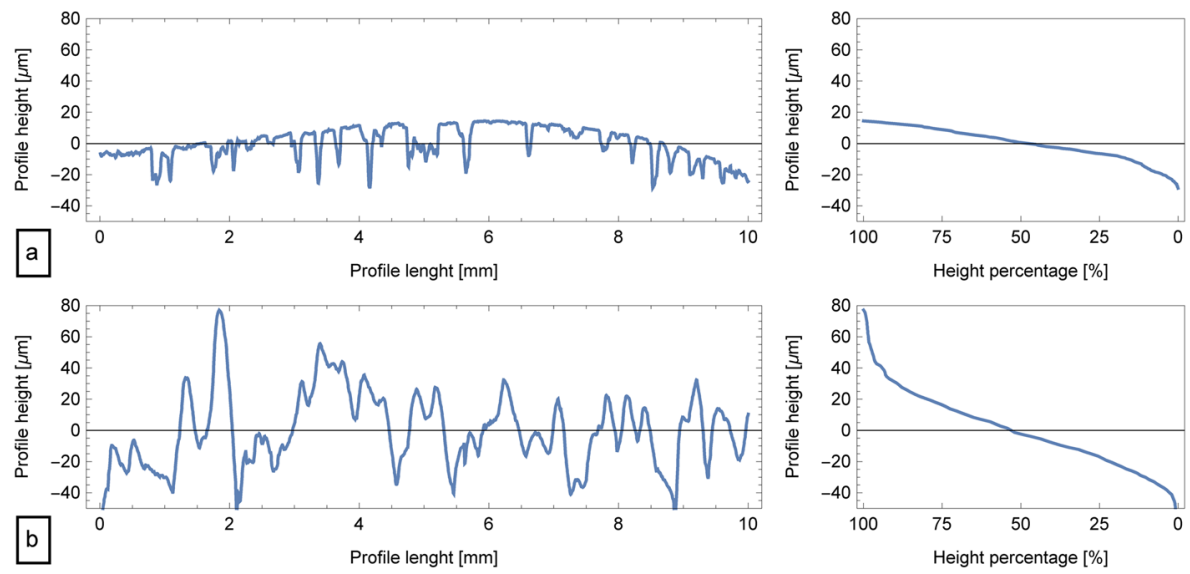

Fig. 5 Sand-blasted (top panel) and manual-grinded (bottom panel) unfiltered profiles

The loss in planarity is more evident if a 3D map of the surface is studied. Figure 6 shows a sand-blasted surface characterized by a homogeneous behavior: this underlines that a possible scattering observed on a surface conditioned by secondary operations probably does not depend upon the SLM fabrication. The grinded surface shows a double curved trend indicating a more complex deformation of the surface. This loss of shape could reduce the improvement obtained by the finishing, and thus must be taken into account.

\section{Tests}

The angular and frequency responses of the horns have been characterized in a custom microwave anechoic chamber, with effective working volume $(l \times w \times h)$ $80 \times 80 \times 80 \mathrm{~cm}^{3}$ (see Fig. 7 for a sketch). Given the apertures of the horns and the wavelengths of interest, the size of this chamber is sufficient to perform tests in the far-field region of the horns.

The test facility is composed of an Anritsu E37247D Vector Network Analyzer (VNA), which can work in the frequency range $[0.04,20] \mathrm{GHz}$, either in frequency sweep or in continuous wave frequency configuration, can send probes in the power range $[-20,10] \mathrm{dBm}$, and has a $\sim 80 \mathrm{~dB}$ dynamic range. This VNA is equipped with the Anritsu 3738A module, which extends the working frequency range to [60, 115] $\mathrm{GHz}$. This setup is completed by a rotary stage equipped with a remotely controlled stepping motor, allowing to sweep the azimuthal angle between the two ports across a $\sim 64^{\circ}$ range. Given the size of the chamber and of the transmitting/receiving modules of the VNA, this system allows measurement and collection of azimuthal cuts of the far-field radiation patterns of the Antenna Under Test (AUT).

The SLM-fabricated AUT was coupled to port 1, while port 2 was swept at constant angular velocity around the nominal position of the phase center of the AUT. As long as the optical alignment between the two ports is correct (i.e., the horn at 

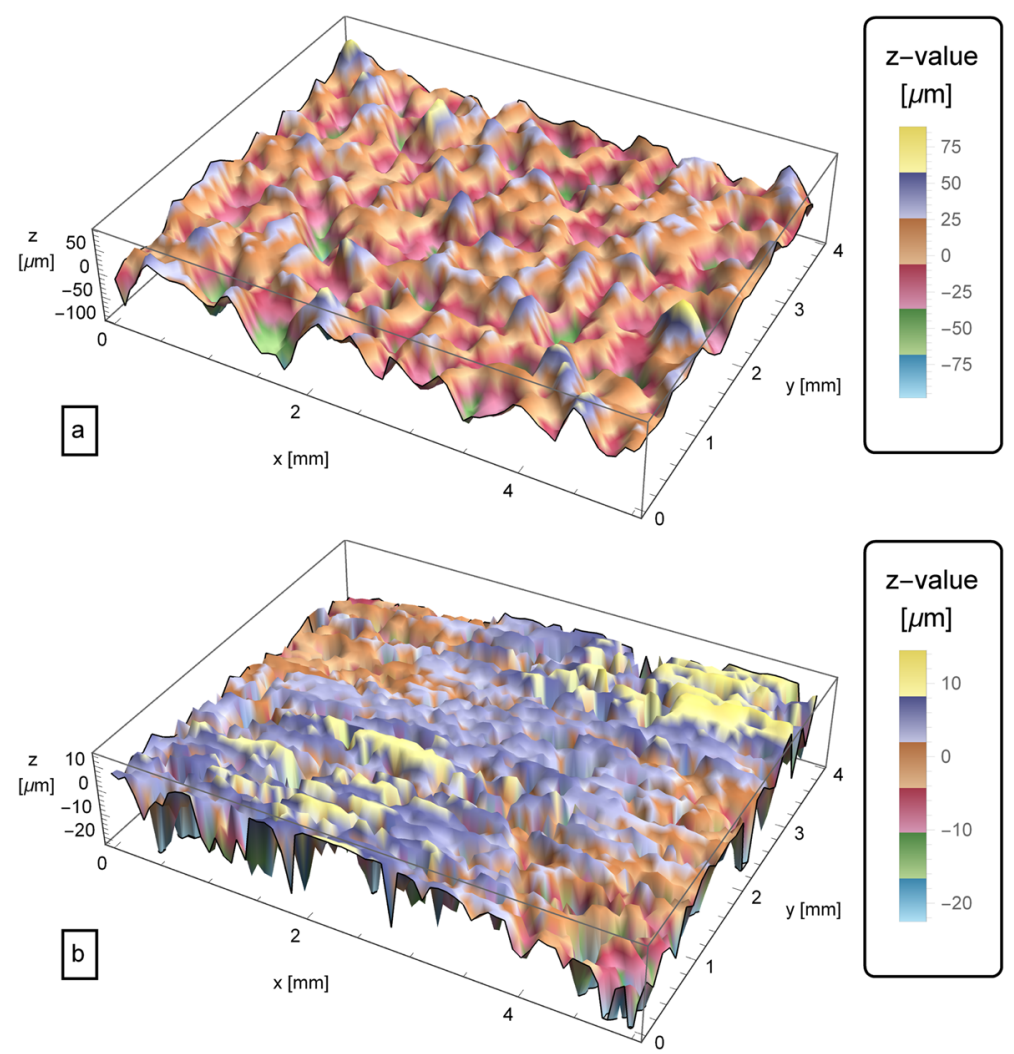

Fig. 6 Sand-blasted (left panel) and manual-grinded (right panel) 3D maps

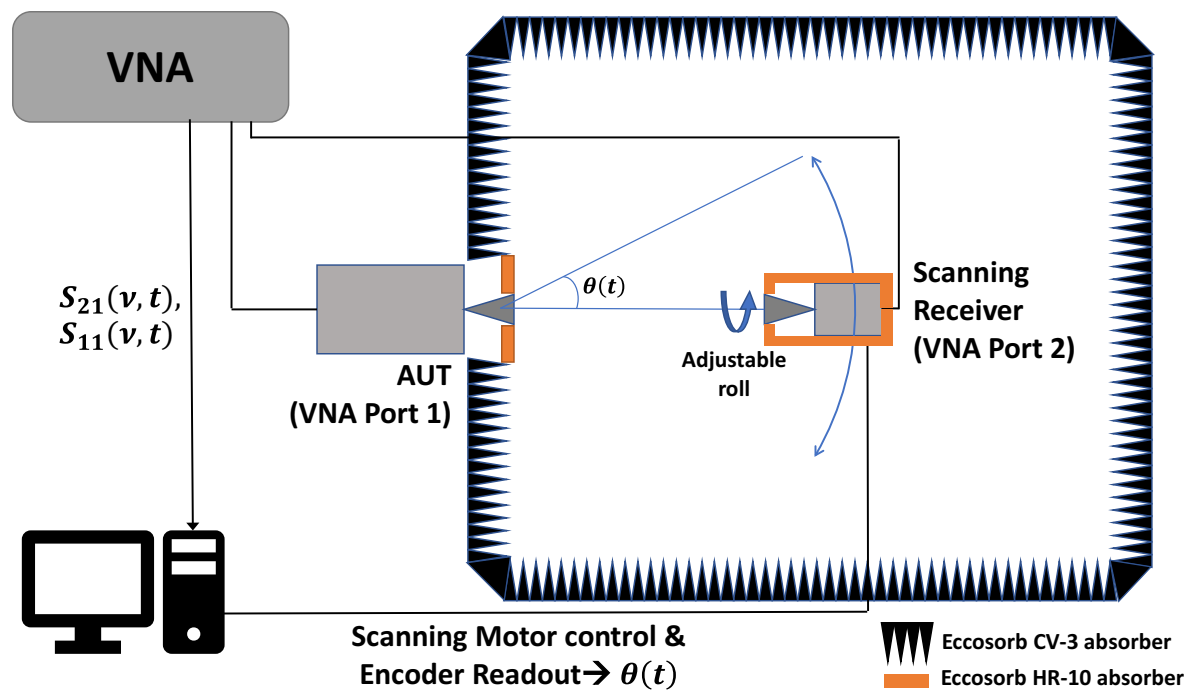

Fig. 7 Sketch of the experimental setup 
port 2 is swept in azimuth while pointing at the phase center of the horn at port 1), the details of the radiation pattern of the horn at port 2 are not relevant, and its onaxis gain determines only the overall coupling efficiency of the system, thus allowing to measure the radiation pattern across the H-plane of the AUT by simply recording the $S_{21}$ signal as a function of the azimuthal angle. A second, manually operated, rotary stage allows rotating the receiver at port 2 by an arbitrary roll angle. A $90^{\circ}$ roll with respect to the orientation that maximizes the $S_{21}$ in the on-axis position allows performing an H-plane cross-polar scan.

The present setup does not allow a full 3D mapping of the radiation pattern, but the commercially procured, gold-plated copper horn has been used as a reference, and its measured performance compared with the ones measured for the SLM-manufactured samples. The impact of the different material, of the manufacturing process, and of the post-processing techniques can therefore be assessed by quantifying the performance degradation with respect to the reference horn which the SLM samples are meant to mimic.

\section{Results}

A preliminary set of measurements with the above setup was performed with the aim of characterizing the overall quality of the assembly and of the optical coupling between the two VNA ports, and to record a reference dataset where the AUT is the commercial horn.

As a first step, the $S_{11}$ co-polar response was used to quantify the level of mismatch between the two ports in the setup. In particular, its spatial modulation as a function of the scan angle tracks a plethora of degenerate systematic effects (tilts, offsets, scan miscentering, etc.) such that the scan is performed over a surface which does not follow the ideal shape of the return wavefront. The measured $S_{11}$ profile after alignment and adjustments is shown in the bottom panel of Fig. 8. The maximum observed peak-to-peak fluctuation in this profile was narrowed to $\sim 0.6 \mathrm{~dB}$ in the main beam region, and less than $0.15 \mathrm{~dB}$ in the far sidelobe region. These levels set also the expected narrowband level of systematic uncertainty over the characterization of the samples.

The performance of the reference horn was then measured across the whole Wband, from 75 to $110 \mathrm{GHz}$. As an example, the co-polar and cross-polar radiation patterns are shown in Fig. 8 for a frequency of $90 \mathrm{GHz}$. The peak gain at this frequency is $22.7 \mathrm{dBi}$, while it ranges from 21.4 to $23.7 \mathrm{dBi}$ from the lower to the upper edge of the W-band.

By offsetting the reference position of the AUT, the full angular range of the sweep has also been used to sample one side of the azimuthal cuts of the horn response far away from the axis. This angular region is interesting since it is sensitive to large-scale surface features which could be affected by the quality of the SLM samples, yielding potential performance degradation with respect to the directivity of the fabricated devices. The corresponding (co-polar) profile is shown in Fig. 9. 


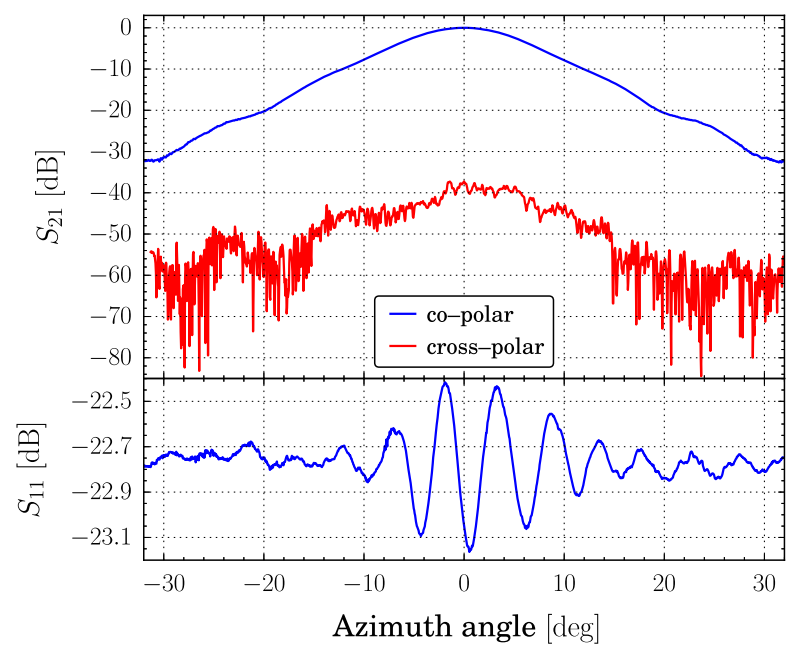

Fig. 8 H-Plane cut of the reference horn radiation pattern, measured at $90 \mathrm{GHz}$

After validating the setup, the reference horn at port 1 was replaced with the SLMmanufactured samples and the azimuthal scans were repeated. The $S_{21}$ results are shown in Figs. 10 and 11.

While some difference at the level of $2 \mathrm{~dB}$ is evident in the radiation patterns at few tens of degrees from the horn axis, it does not hinder the usability of the horn. The peak gains of the two horns are slightly lower than the reference horn, by 0.2 $\mathrm{dB}$ for the sand blasted sample and by $0.5 \mathrm{~dB}$ for the manually grinded one. In order to investigate the physical origin of such excess loss, we performed a finite element simulation of the horn antenna with the Ansys HFSS software with parametric values

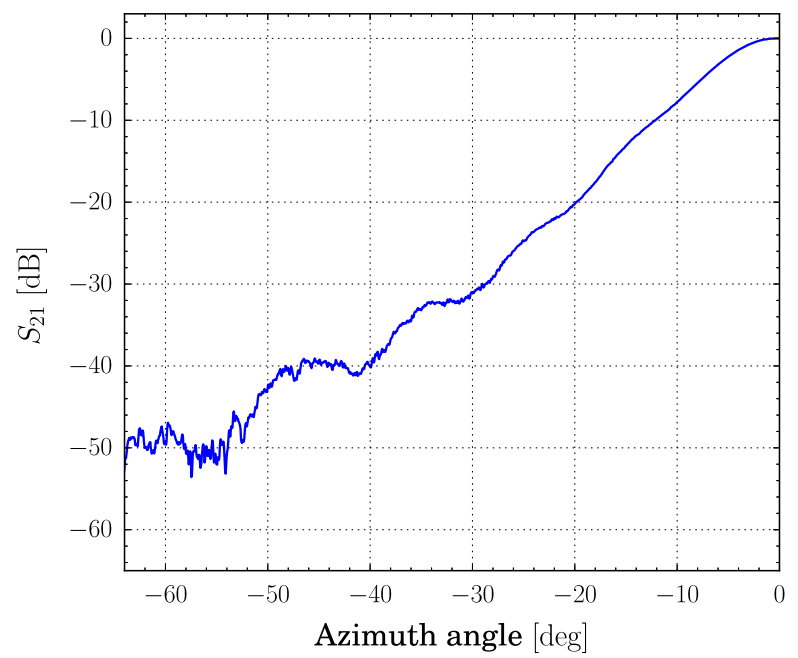

Fig. 9 H-Plane cut of the reference horn radiation pattern, measured at $90 \mathrm{GHz}$ - far sidelobe region 


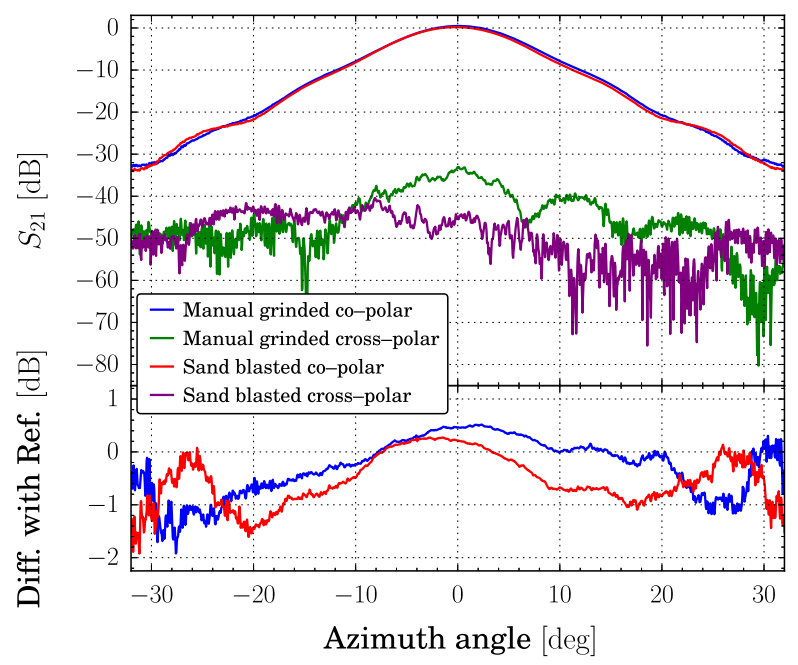

Fig. $10 S_{21}$ profile at $90 \mathrm{GHz}$ for the two SLM samples. Color coding is used to discriminate the two post-processing strategies. Top panel Absolute co-polar and cross-polar patterns. Bottom panel Deviation of the measured co-polar signals from the reference profile

for the material conductivity. We find that the difference in surface conductivity, due both to different material and surface roughness of the horn walls, can account at most for $20 \%$ of the measured loss. While the measured effect needs to be further investigated with new samples, we here stress that both the post-processing methods used on the SLM horns leave the waveguide section substantially untouched (and unfinished) with respect to the horn walls. As suggested also in [29], the waveguide section can be the most significant and hard to model source of additional loss for

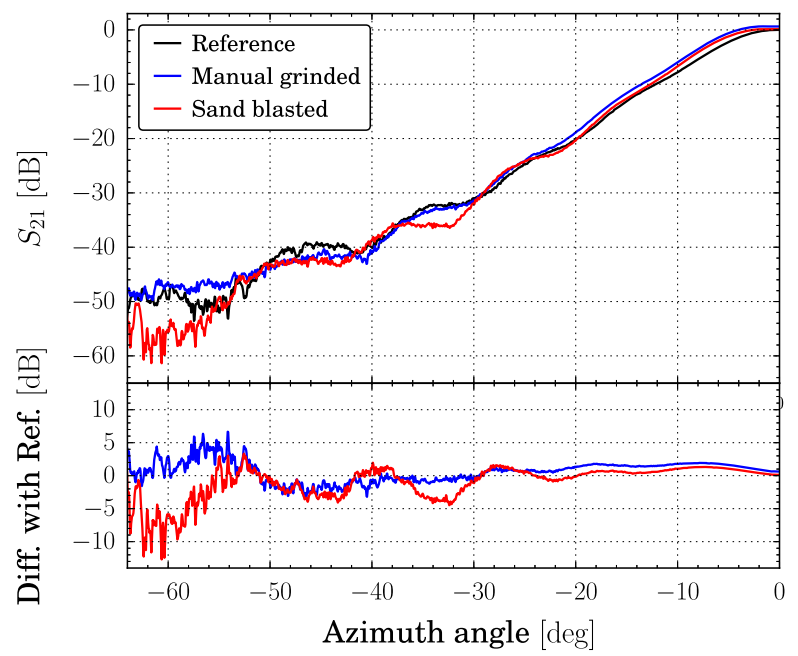

Fig. 11 Extended range 90-GHz scan 
these devices: losses in WR10 waveguides built by direct metal printing have been measured in the range $0.02-0.7 \mathrm{~dB} / \mathrm{mm}$ because of roughness [10], so that the $3.8-\mathrm{mm}$ waveguide in our samples remains the section where most of the loss takes place.

The cross-polar response along the H-plane was also characterized with the same setup: since the original horn is not optimized for low cross-polar response, its absolute cross-polar performance is not outstanding by any means. Anyway, both the SLM manufactured samples exhibit an overall comparable level of cross-polarization. The measured profile for the sand-blasted sample exhibits a clear asymmetry with respect to the main axis, which could be easily attributed to a large-scale, uneven deformation of the internal surface due to the sand-blasting procedure, or to an inhomogeneous surface finish intrinsic to the process (as already shown from the measurements described in Section 4).

In order to check the stability of the setup and the presence of systematics in the placement and assembly of the AUTs, we also show in Fig. 12 the $S_{11}$ measurements of the two SLM samples. The shapes of the observed patterns do show some deviation from those of the original horn (bottom panel of Fig. 8), consistently with the deviations in the $S_{21}$ patterns, but they remain at the same peak-to-peak levels of the reference horn, so that an overall systematic uncertainty of $\pm 1 \mathrm{~dB}$ in the main lobe region can be safely assumed with this setup.

Once again, no significant preference with respect to performance can be expressed for the two types of surface post-processing.

Finally, we show in Fig. 13 the measured frequency dependence of the S parameters of the SLM samples across the whole W-band, on-axis, and at 6 azimuth angles spanning a $30^{\circ}$ range away from system axis. Given the uncertainties quoted above, the frequency responses are quite consistent with that of the reference horn within $10^{\circ}$ from the axis, corresponding to the main lobe of the radiation pattern. At higher

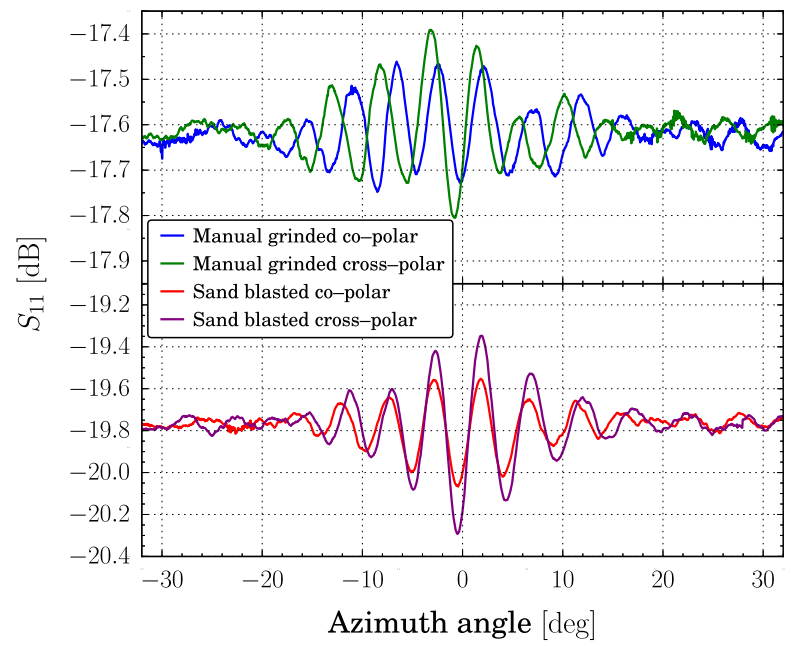

Fig. $12 S_{11}$ co-polar and cross-polar profiles at $90 \mathrm{GHz}$ for the two SLM samples. Color coding is used to discriminate the two post-processing strategies. Top panel Manually grinded sample. Bottom panel Sand-blasted sample 


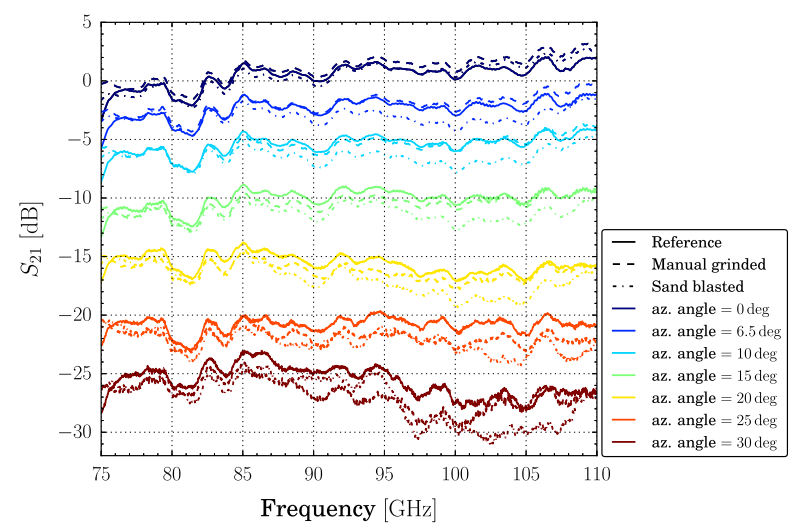

Fig. 13 Frequency dependence of $S_{21}$. Color codes correspond to different angles from the axis. Solid, dashed, and dot-dashed line styles identify the different horn samples. All curves share the same normalization, which was arbitrarily chosen to be the $S_{21}$ amplitude at $90 \mathrm{GHz}$ of the reference horn

azimuths, however, a systematic degradation of performance with a slight increase of the deviation at the top edge of the band can be observed. This behavior is expected from the overall higher loss due to the surface roughness of the SLM samples, and certainly demands a more careful designation of the post-processing steps to ensure higher frequency usability of such devices, especially if long and complex waveguide structures are integrated in the design.

\section{Conclusion}

Based on radiation pattern and gain measurements and on their characterization across the W-band, we can conclude that acceptable performance of additivemanufactured, Al-based horn antennas can be achieved after the application of very basic post-processing techniques. Given the current level of robustness and the reproducibility of the test setup, both the azimuthal patterns of the co-polar response and their degradation at frequencies close to the top edge of the band $(110 \mathrm{GHz})$ show a good consistency with the behavior of the reference commercial sample. Peak gain values and gain variation over the main beam region of the radiation pattern are consistent within $\sim 0.5 \mathrm{~dB}$. We speculate that residual surface roughness in the waveguide section of the tested assemblies may be responsible for most of this loss. Our results are consistent with a similar study performed in [29] with smooth conical horns fabricated with SLM of Cu-15Sn powder. In addition, [10] find similar criticalities related to the roughness waveguide section and general fabrication tolerance when testing W-band corrugated feedhorns fabricated through AM of conductive polymers.

No significant quantitative result allows us to express a definite preference for the manual grinding or for the sand-blasting post-processing procedures. Anyway, based on the surface characterization results, the former appears to yield highly unpredictable levels of large-scale uniformity, which could in principle average into 
significant (although not evident for the individual case discussed here) effect on the electromagnetic performance of the horns. For this reason, and in view of mass production of such devices, it is safe to assume that future development of Al-based SLM horns for millimeter wavelengths will have to rely on customized post-processing steps with a high degree of reproducibility, and possibly higher capacity to deal with the roughness of the smallest waveguide sections of the manufactured devices.

Funding Open access funding provided by Università degli Studi di Roma La Sapienza within the CRUICARE Agreement.

Open Access This article is licensed under a Creative Commons Attribution 4.0 International License, which permits use, sharing, adaptation, distribution and reproduction in any medium or format, as long as you give appropriate credit to the original author(s) and the source, provide a link to the Creative Commons licence, and indicate if changes were made. The images or other third party material in this article are included in the article's Creative Commons licence, unless indicated otherwise in a credit line to the material. If material is not included in the article's Creative Commons licence and your intended use is not permitted by statutory regulation or exceeds the permitted use, you will need to obtain permission directly from the copyright holder. To view a copy of this licence, visit http://creativecommonshorg/licenses/by/4.0/.

\section{References}

1. Addamo, G., Peverini, O.A., Manfredi, D., Calignano, F., Paonessa, F., Virone, G., Tascone, R., Dassano, G.: Additive manufacturing of ka-band dual-polarization waveguide components. IEEE Transactions on Microwave Theory and Techniques 66(8), 3589-3596 (2018).

2. Bandyopadhyay, A., Bose, S.: Additive Manufacturing. CRC Press (2015). https://www.crcpress.com/ Additive-Manufacturing/Bandyopadhyay-Bose/p/book/9781482223590.

3. Boschetto, A., Bottini, L., Veniali, F.: Surface roughness and radiusing of ti6al4v selective laser melting-manufactured parts conditioned by barrel finishing. The International Journal of Advanced Manufacturing Technology 94(5), 2773-2790 (2018). https://doi.org/10.1007/s00170-017-1059-6.

4. Campbell, J.: Castings. Butterworth-Heinemann (2003). https:/www.elsevier.com/books/castings/ campbel1/978-0-7506-4790-8.

5. Childs, T.H.C., Hauser, C.: Raster scan selective laser melting of the surface layer of a tool steel powder bed. Proceedings of the Institution of Mechanical Engineers, Part B: Journal of Engineering Manufacture 219(4), 379-384 (2005). https://doi.org/10.1243/095440505X32201.

6. Chio, T., Huang, G., Zhou, S.: Application of direct metal laser sintering to waveguide-based passive microwave components, antennas, and antenna arrays. Proceedings of the IEEE 105(4), 632-644 (2017).

7. Chua, C.K., Leong, K.F., Lim, C.S.: Rapid prototyping: principles and applications. World Scientific (2010). https://www.worldcat.org/title/rapid-prototyping-principles-and-applications/oclc/ 403480284.

8. Columbro, F., Madonia, P.G., Lamagna, L., Battistelli, E.S., Coppolecchia, A., de Bernardis, P., Gualtieri, R., Masi, S., Paiella, A., Piacentini, F., Presta, G., Biasotti, M., D’Alessandro, G., Gatti, F., Mele, L., Siri, B.: Swipe multi-mode pixel assembly design and beam pattern measurements at cryogenic temperature. Journal of Low Temperature Physics 199(1), 312-319 (2020). https://doi.org/10.1007/s10909-020-02396-4.

9. Coppolecchia, A., Paiella, A., Lamagna, L., Presta, G., Battistelli, E.S., de Bernardis, P., Castellano, M.G., Columbro, F., Masi, S., Mele, L., Pettinari, G., Piacentini, F.: W-band lumped element kinetic inductance detector array for large ground-based telescopes. Journal of Low Temperature Physics 199(1), 130-137 (2020). https://doi.org/10.1007/s10909-019-02275-7.

10. Decrossas, E., Reck, T., Lee, C., Jung-Kubiak, C., Mehdi, I., Chattopadhyay, G.: Evaluation of $3 d$ printing technology for corrugated horn antenna manufacturing. In: 2016 IEEE International Symposium on Electromagnetic Compatibility (EMC), pp. 251-255 (2016).

11. EOS GmbH-Electro Optical Systems: Material data sheet EOS Aluminum AlSi10Mg (2014). 
12. Gadelmawla, E., Koura, M., Maksoud, T., Elewa, I., Soliman, H.: Roughness parameters. Journal of Materials Processing Technology 123, 133-145 (2002). https://doi.org/10.1016/S0924-0136(02)00 060-2.

13. Kumar, S.: 10.05 - selective laser sintering/melting. In: S. Hashmi, G.F. Batalha, C.J.V. Tyne, B. Yilbas (eds.) Comprehensive Materials Processing, pp. 93 - 134. Elsevier, Oxford (2014). https://doi.org/10.1016/B978-0-08-096532-1.01003-7. http://www.sciencedirect.com/science/article/ pii/B9780080965321010037.

14. Lamagna, L., Addamo, G., Ade, P.A.R., Baccigalupi, C., Baldini, A.M., Battaglia, P.M., Battistelli, E., Baù, A., Bersanelli, M., Biasotti, M., Boragno, C., Boscaleri, A., Caccianiga, B., Caprioli, S., Cavaliere, F., Cei, F., Cleary, K.A., Columbro, F., Coppi, G., Coppolecchia, A., Corsini, D., Cuttaia, F., D’Alessandro, G., de Bernardis, P., De Gasperis, G., De Petris, M., Torto, F.D., Fafone, V., Farooqui, Z., Farsian, F., Fontanelli, F., Franceschet, C., Gaier, T.C., Gatti, F., Genova-Santos, R., Gervasi, M., Ghigna, T., Grassi, M., Grosso, D., Incardona, F., Jones, M., Kangaslahti, P., Krachmalnicoff, N., Mainini, R., Maino, D., Mandelli, S., Maris, M., Masi, S., Matarrese, S., May, A., Mena, P., Mennella, A., Molina, R., Molinari, D., Morgante, G., Nati, F., Natoli, P., Pagano, L., Paiella, A., Paonessa, F., Passerini, A., Perez-de Taoro, M., Peverini, O.A., Pezzotta, F., Piacentini, F., Piccirillo, L., Pisano, G., Polastri, L., Polenta, G., Poletti, D., Presta, G., Realini, S., Reyes, N., Rocchi, A., Rubino-Martin, J.A., Sandri, M., Sartor, S., Schillaci, A., Signorelli, G., Soria, M., Spinella, F., Tapia, V., Tartari, A., Taylor, A., Terenzi, L., Tomasi, M., Tommasi, E., Tucker, C., Vaccaro, D., Vigano, D.M., Villa, F., Virone, G., Vittorio, N., Volpe, A., Watkins, B., Zacchei, A., Zannoni, M.: Progress report on the large-scale polarization explorer. Journal of Low Temperature Physics (2020). https://doi.org/10.1007/s10909-020-02454-X.

15. Lomakin, K., Pavlenko, T., Ankenbrand, M., Sippel, M., Ringel, J., Scheetz, M., Klemm, T., Gräf, D., Helmreich, K., Franke, J., Gold, G.: Evaluation and characterization of 3-d printed pyramid horn antennas utilizing different deposition techniques for conductive material. IEEE Transactions on Components, Packaging and Manufacturing Technology 8(11), 1998-2006 (2018).

16. Masi, Silvia, Coppolecchia, A., Battistelli, E., de Bernardis, P., Columbro, F., D’Alessandro, G., Lamagna, L., Paiella, A., Piacentini, F., Presta, G.: Balloon-borne cosmic microwave background experiments. EPJ Web Conf. 209, 01046 (2019). https://doi.org/10.1051/epjconf/201920901046.

17. Paiella, A., Coppolecchia, A., Castellano, M.G., Colantoni, I., Cruciani, A., D'Addabbo, A., de Bernardis, P., Masi, S., Presta, G.: Development of lumped element kinetic inductance detectors for the w-band. Journal of Low Temperature Physics 184(1), 97-102 (2016). https://doi.org/10.1007/s10909-015-1470-z.

18. Paiella, A., Coppolecchia, A., Lamagna, L., Ade, P., Battistelli, E., Castellano, M.G., Colantoni, I., Columbro, F., D’Alessandro, G., de Bernardis, P., Gordon, S., Masi, S., Mauskopf, P., Pettinari, G., Piacentini, F., Pisano, G., Presta, G., Tucker, C.: Kinetic inductance detectors for the OLIMPO experiment: design and pre-flight characterization. Journal of Cosmology and Astroparticle Physics 2019(01), 039-039 (2019). https://doi.org/10.1088/1475-7516/2019/01/039.

19. Peverini, O.A., Lumia, M., Calignano, F., Addamo, G., Lorusso, M., Ambrosio, E.P., Manfredi, D., Virone, G.: Selective laser melting manufacturing of microwave waveguide devices. Proceedings of the IEEE 105, 620 - 631 (2017). https://doi.org/10.1109/JPROC.2016.2620148.

20. Peverini, O.A., M.Lumia, Addamo, G., Paonessa, F., Virone, G., Tascone, R., Calignano, F., Cattano, G., Manfredi, D.: Integration of an h-plane bend, a twist, and a filter in $k_{u} / k$-band through additive manufacturing. IEEE Transactions on Microwave Theory and Technique 66, 2210 - 2219 (2018). https://doi.org/10.1109/TMTT.2018.2809505.

21. Trappe, N., Bucher, M., Bernardis, P.D., Delabrouille, J., Deo, P., DePetris, M., Doherty, S., Ghribi, A., Gradziel, M., Kuzmin, L., Maffei, B., Mahashabde, S., Masi, S., Murphy, J.A., Noviello, F., O’Sullivan, C., Pagano, L., Piacentini, F., Piat, M., Pisano, G., Robinson, M., Stompor, R., Tartari, A., van der Vorst, M., Verhoeve, P.: Next generation sub-millimeter wave focal plane array coupling concepts: an ESA TRP project to develop multichroic focal plane pixels for future CMB polarization experiments. In: W.S. Holland, J. Zmuidzinas (eds.) Millimeter, Submillimeter, and Far-Infrared Detectors and Instrumentation for Astronomy VIII, vol. 9914, pp. 236 - 242. International Society for Optics and Photonics, SPIE (2016). https://doi.org/10.1117/12.2231267.

22. Vv.Aa.: ISO 4288:1996. Geometrical Product Specifications (GPS) - Surface texture: Profile method - Rules and procedures for the assessment of surface texture (1996).

23. Vv.Aa.: ISO 4287:1997. Geometrical Product Specification (GPS)-Surface Texture: Profile method Terms, Definition and Surface Texture Parameters (1997). 
24. Vv.Aa.: Geometrical Product Specifications (GPS)-Filtration-Part 22:Linear Profile Filters: Spline Filters (2015).

25. Vv.Aa.: ISO/ASTM52900-15, Standard Terminology for Additive Manufacturing-General Principles-Terminology. ASTM International (2015).

26. Vv.Aa.: ISO 6892-1:2016 Metallic materials - Tensile testing - Part 1: Method of test at room temperature (2016).

27. Whitehouse, D.: Handbook of Surface Metrology. CRC Press (1994).

28. Wohlers, T.T., Caffrey, T.: Wohlers Report 2015: 3D Printing and Additive Manufacturing State of the Industry Annual Worldwide Progress Report. Wohlers Associates (2015). https://www.worldcat.org/title/wohlers-report-2015-3d-printin g-and-additive-manufacturing-state-ofthe-industry-annual-worldwide-progress-r eport/oclc/908262396?referer=di\&ht=edition.

29. Zhang, B., Zhan, Z., Cao, Y., Gulan, H., Linnér, P., Sun, J., Zwick, T., Zirath, H.: Metallic 3-d printed antennas for millimeter- and submillimeter wave applications. IEEE Transactions on Terahertz Science and Technology 6(4), 592-600 (2016). https://doi.org/10.1109/TTHZ.2016.2562508.

30. Zhang, B., Zirath, H.: Metallic 3-d printed rectangular waveguides for millimeter-wave applications. IEEE Transactions on Components, Packaging and Manufacturing Technology 6(5), 796-804 (2016). https://doi.org/10.1109/TCPMT.2016.2550483.

Publisher's Note Springer Nature remains neutral with regard to jurisdictional claims in published maps and institutional affiliations. 